\title{
Measuring carbon nanotube vibrations using a single-electron transistor as a fast linear amplifier
}

Cite as: Appl. Phys. Lett. 113, 153101 (2018); https://doi.org/10.1063/1.5052185

Submitted: 14 August 2018. Accepted: 26 September 2018 . Published Online: 09 October 2018

Yutian Wen, N. Ares (D., T. Pei, G. A. D. Briggs (D), and E. A. Laird (iD)

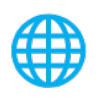

\section{ARTICLES YOU MAY BE INTERESTED IN}

Improving the read-out of the resonance frequency of nanotube mechanical resonators Applied Physics Letters 113, 063104 (2018); https://doi.org/10.1063/1.5045309

Gate-tunable graphene-organic interface barrier for vertical transistor and logic inverter Applied Physics Letters 113, 153301 (2018); https://doi.org/10.1063/1.5045497

Graphene-based positron charge sensor

Applied Physics Letters 113, 154101 (2018); https://doi.org/10.1063/1.5053477

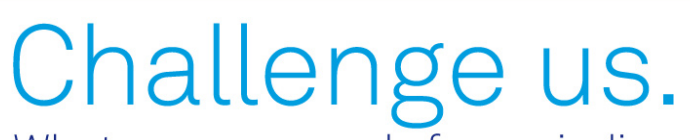
What are your needs for periodic signal detection?

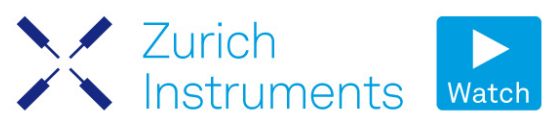

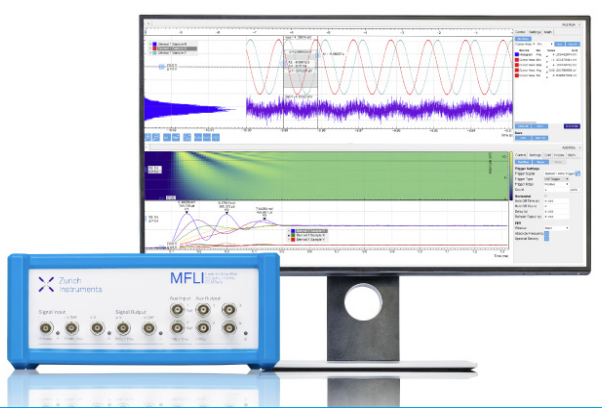

Appl. Phys. Lett. 113, 153101 (2018); https://doi.org/10.1063/1.5052185

113, 153101 


\title{
Measuring carbon nanotube vibrations using a single-electron transistor as a fast linear amplifier
}

\author{
Yutian Wen, ${ }^{1}$ N. Ares, ${ }^{1}$ T. Pei, ${ }^{1}$ G. A. D. Briggs, ${ }^{1}$ and E. A. Laird ${ }^{1,2, a)}$ \\ ${ }^{1}$ Department of Materials, University of Oxford, Parks Road, Oxford OXI 3PH, United Kingdom \\ ${ }^{2}$ Department of Physics, Lancaster University, Lancaster LA1 4YB, United Kingdom
}

(Received 14 August 2018; accepted 26 September 2018; published online 9 October 2018)

\begin{abstract}
We demonstrate sensitive and fast electrical measurements of a carbon nanotube mechanical resonator. The nanotube is configured as a single-electron transistor, whose conductance is a sensitive transducer for its own displacement. Using an impedance-matching circuit followed by a cryogenic amplifier, the vibrations can be monitored at radio frequency. The sensitivity of this continuous displacement measurement approaches within a factor 470 of the standard quantum limit. Published by AIP Publishing. https://doi.org/10.1063/1.5052185
\end{abstract}

Suspended carbon nanotubes are mechanical resonators ${ }^{1}$ with low mass, high compliance, and high quality factor, ${ }^{2,3}$ which make them sensitive electromechanical detectors for tiny forces ${ }^{4}$ and masses. ${ }^{5-7}$ The time-averaged current through a vibrating nanotube probe electron-phonon coupling, ${ }^{8-11}$ nonlinear dissipation, ${ }^{12}$ and mechanical mode mixing ${ }^{13}$ on the nanoscale. Time-resolved measurements go further, allowing for the study of transient effects such as spin switching, ${ }^{14,15}$ mechanical dephasing, ${ }^{16}$ or even force-detected magnetic resonance. ${ }^{17}$ Although the low mass favors large electromechanical coupling, the large electrical impedance of nanotube devices makes it difficult to amplify the current signal with high sensitivity and bandwidth, especially since low temperatures are needed to suppress thermal noise.

One approach is to downconvert the electromechanical signal to a frequency within the bandwidth of a cryogenic current amplifier, using either two-source mixing or the non-linear conductance of the nanotube itself. ${ }^{18,19}$ For fast measurements, parasitic capacitance must be minimised by placing the amplifier close to the resonator. The resulting heat load has usually prevented operation below $1 \mathrm{~K},{ }^{16,19}$ although recently such a setup achieved high sensitivity at millikelvin temperatures and with a bandwidth of $87 \mathrm{kHz}^{20}$ A second approach, with a higher bandwidth, is to detect the changing capacitance between the vibrating nanotube and a pickup antenna. ${ }^{21}$ However, the small size of the pickup antenna means that a large electric field is needed to generate an appreciable signal. A third approach, used for other kinds of nanoscale resonators, ${ }^{22,23}$ is to connect the resonator's output directly to a fast amplifier matched to the cable impedance (typically $50 \Omega$ ). However, the electrical divider formed between the large impedance of the device and the small impedance of the cable degrades the signal.

Here, we demonstrate a circuit that combines sensitivity with high speed by monitoring the electromechanical signal directly while requiring only a DC bias voltage. The circuit exploits a single-electron transistor (SET) defined within the nanotube as the initial stage of displacement amplification. ${ }^{2,9-11,24-27}$ The SET output current, which depends linearly on displacement, is monitored directly at radio

${ }^{a)}$ Electronic mail: e.a.laird@lancaster.ac.uk frequency using a low-noise cryogenic radio-frequency (RF) amplifier with a $\mathrm{MHz}$ bandwidth. To improve the coupling between the SET and the cryogenic amplifier, we use an impedance-matching stage based on a tunable RF tank circuit. $^{28}$ We characterize the displacement imprecision achieved in this setup and show that it approaches within a factor 470 of the limit set by quantum mechanics. This technique combines the speed allowed by the RF readout with the high sensitivity of an SET amplifier integrated into the moving nanotube.

The experimental setup is shown in Fig. 1(a). The resonator is fabricated by stamping a carbon nanotube across lithographically patterned $\mathrm{Cr} / \mathrm{Au}$ contact electrodes, giving a suspended length of $l=800 \mathrm{~nm} .{ }^{29-31}$ Beneath the nanotube, five predefined $\mathrm{Cr} / \mathrm{Au}$ finger gates, labelled G1-5, allow tuning of the electrical potential along the nanotube. This device is bonded to a printed circuit board and loaded into a $25 \mathrm{mK}$ dilution refrigerator. For electromechanical excitation, gate G2 is connected via a bias tee to an RF drive line [port 1 in Fig. 1(a)] and is driven either by a RF signal generator or using the output of a network analyzer.

With the drain electrode held at ground, a source-drain bias $V_{\mathrm{SD}}$, applied to the source electrode, drives a current through the nanotube which can be measured at both DC and RF. The DC current $I_{\mathrm{DC}}$ is monitored using a roomtemperature current amplifier connected to the drain. The RF response is measured using a cryogenic amplifier anchored at $5 \mathrm{~K}$ inside the cryostat. A resonant tank circuit [Fig. 1(a) right], constructed using chip inductors and capacitors on the circuit board and bonded to the source electrode, is interposed between the device and the amplifier to transduce the RF current through the nanotube to an output voltage $V_{\text {out }}$, which is fed to the cryogenic amplifier. ${ }^{21}$ This amplified signal is fed via port 2 of the cryostat into the network analyzer. The tank circuit incorporates a variable capacitor $C_{\mathrm{S}}$, controlled with a tuning voltage $V_{\mathrm{S}}$, which adjusts the electrical resonance frequency. ${ }^{28}$

To allow sensitive electromechanical measurements, the suspended nanotube is operated as an SET. This SET is formed between the Schottky barriers along the nanotube and tuned using DC voltages applied to the finger gates. ${ }^{32,33}$ Figure 1(b) plots the DC current $I_{\mathrm{DC}}$ through the nanotube as 

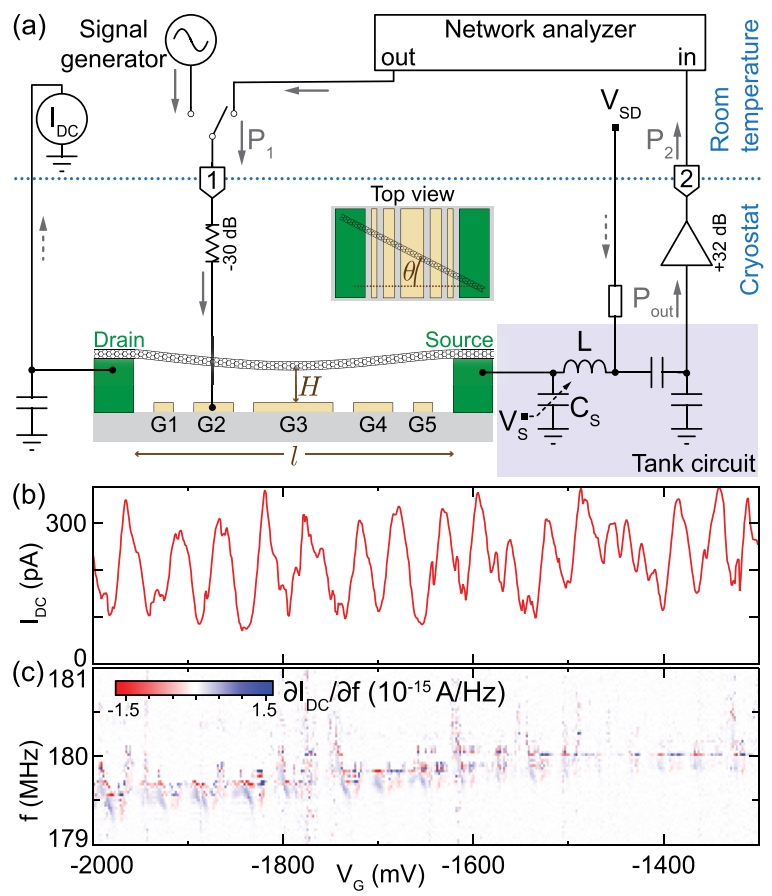

FIG. 1. (a) Device and measurement setup. The vibrating nanotube is suspended between source and drain electrodes and over five gate electrodes that define a quantum dot potential. Gate 2 is connected to an attenuated high-frequency line for mechanical actuation. The device is biased by voltage $V_{\mathrm{SD}}$ and measured via both DC transport and a tunable RF circuit (see the text). The DC current path is marked by dashed arrows and the RF path by solid arrows. The length $l$ and suspended height $H$ of the nanotube are indicated in the main diagram, and the inset marks the alignment angle $\theta$. (b) Coulomb blockade peaks measured in DC current as a function of DC voltage $V_{\mathrm{G}}$ on gate 2 , with $V_{\mathrm{SD}}=5 \mathrm{mV}$. (c) Characterization of the mechanical resonance in transport. With RF power applied at frequency $f$, the derivative $\partial I_{\mathrm{DC}} / \partial f$ shows the resonance as a weakly gate-dependent feature at $f \sim 180 \mathrm{MHz}$. The RF power at port 1 was $P_{1}=-35 \mathrm{dBm}$, and the bias was $V_{\mathrm{SD}}=5 \mathrm{mV}$. In (b) and (c), the other gate voltages were held at positive voltages between 0 and $300 \mathrm{mV}$.

a function of the DC voltage $V_{\mathrm{G}}$ applied to gate G2. The pattern of current peaks indicates Coulomb blockade, confirming the SET behavior.

We first identify the mechanical resonance using DC transport. With an RF drive at frequency $f$ applied to gate $\mathrm{G} 2$, Fig. 1(c) shows the derivative $\partial I_{\mathrm{DC}} / \partial f$ as a function of $f$ and $V_{\mathrm{G}}$. The current has a sharp peak or $\operatorname{dip}$ when $f$ matches the mechanical resonance frequency $f_{\mathrm{M}}$, leading to a feature in the derivative ${ }^{1,2}$ appearing around $f_{\mathrm{M}} \approx 180 \mathrm{MHz}$. The resonance frequency increases with increasing $V_{\mathrm{G}}$ as the mechanical tension changes. It is also modulated by Coulomb blockade because electron tunnelling modifies the effective spring constant. 9,10

We now turn to RF measurements. To optimise the sensitivity, we first tune the tank circuit's electrical resonance frequency $f_{\mathrm{E}}$ to a value near $f_{\mathrm{M}}$. This is inferred from the transmission $S_{21}$ from port 1 to port 2, measured using a network analyzer at different settings of the varactor tuning voltage $V_{\mathrm{S}}$ [Fig. 2(a)]. The main tank circuit resonance is evident as a broad transmission peak, whose frequency increases as $C_{\mathrm{S}}$ is tuned towards lower values. By fitting these traces, we are able to extract the tank circuit parameters (see supplementary material). In the rest of this paper, we fix $V_{\mathrm{S}}=0 \mathrm{~V}$, giving optimum sensitivity around $f_{\mathrm{E}} \approx 178 \mathrm{MHz}$ and a detection bandwidth of $\approx 8 \mathrm{MHz}$, set by the electrical quality factor.

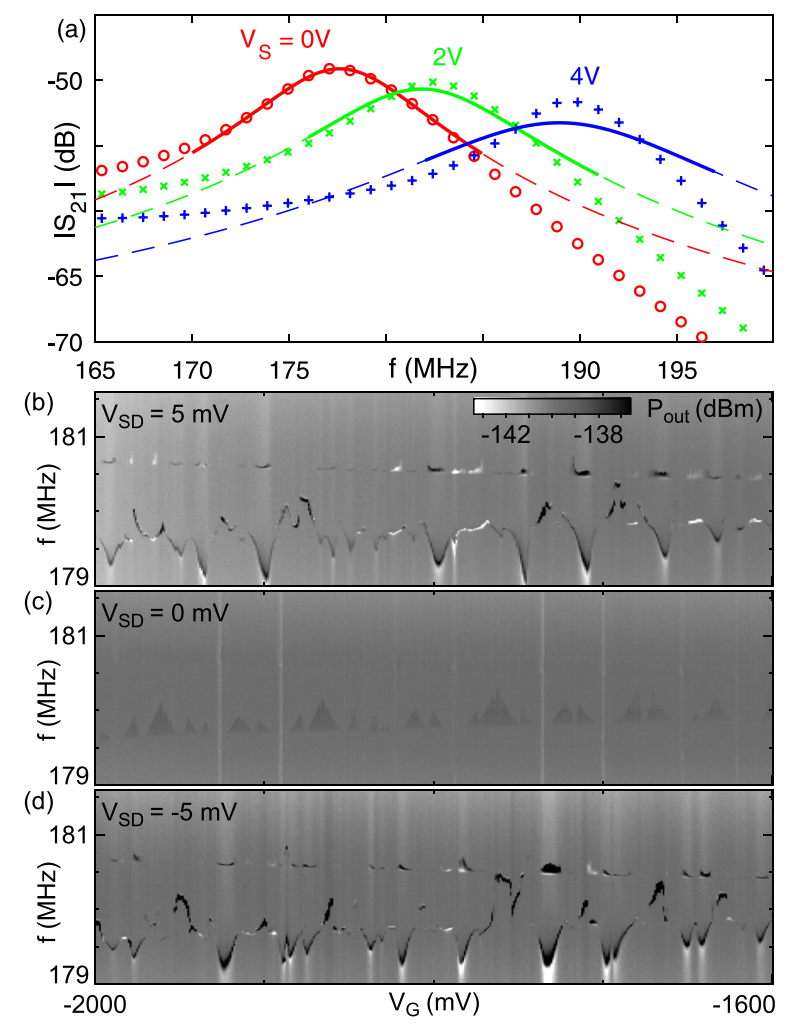

FIG. 2. Characterization of the tank circuit and the mechanical resonance. (a) Electrical transmission from port 1 to port 2, for different settings of the tuning voltage $V_{\mathrm{S}}$ and with $V_{\mathrm{SD}}=0 \mathrm{mV}$. Symbols: data. The main electrical resonance appears as a tunable transmission peak in the range of 178-205 MHz. Curves: Fits to an electrical model of the tank circuit (see supplementary material). The solid section of each curve indicates the fitting range. (b)-(d) Transmitted signal, converted to tank circuit output power $P_{\text {out }}$, as a function of frequency and gate voltage for different settings of $V_{\mathrm{SD}}$. Here, $V_{\mathrm{S}}=0 \mathrm{~V}$ and the drive power is $P_{1}=-70 \mathrm{dBm}$. The main mechanical resonance is evident as a sharp feature in (b) and (d), whose frequency varies with gate voltage because of the Coulomb blockade. A weaker resonance appears at slightly higher frequency. This may be a mode vibrating approximately in the plane of the sample, i.e., orthogonal to the stronger resonance, and is not studied further.

The mechanical signal appears as a sharp resonance superimposed on the electrical transmission peak when a source-drain bias $V_{\mathrm{SD}}$ is applied. This is evident in Figs. 2(b)-2(d), which show the power $P_{\text {out }}$ transmitted from the output of the tank circuit, plotted as a function of frequency and gate voltage. This signal arises because the motion of the nanotube relative to the gates modulates the SET conductance, leading to a current oscillating at the vibration frequency. This current is transduced to an RF output voltage $V_{\text {out }}$, with $P_{\text {out }}=V_{\text {out }}^{2} / Z_{0}$, where $Z_{0}=50 \Omega$ is the line impedance. As expected from this mechanism, the signal appears for both positive bias and negative bias [Figs. 2(b) and 2(d)] but nearly vanishes at zero bias [Fig. 2(c)]. The fact that the signal is larger with a bias applied confirms that it arises mainly from the SET conductance. The weak signal remaining at zero bias indicates a small capacitive contribution. ${ }^{21}$ We interpret this mode as the fundamental out-of-plane flexural mode.

We now characterize the displacement sensitivity. We do this by measuring the signal and the noise of the output voltage when the nanotube is driven to a fixed displacement amplitude, by adding a known oscillating gate voltage $\delta V_{\mathrm{G}}$ to $V_{\mathrm{G}}$. This measurement is performed with the gate voltage 
tuned to the flank of a Coulomb blockade peak [Fig. 3(a)]. As expected, this gate voltage leads to a strong mechanical signal [Fig. 3(b)].

Both signal and noise are extracted from Fig. 3(c), which shows the power spectrum $P_{\text {out }}$, measured with rootmean-square driving amplitude $\delta V_{\mathrm{G}}=1.3 \mu \mathrm{V}$ and for different settings of the network analyzer's resolution bandwidth $\Delta f$. The mechanical resonance is evident at frequency $f_{\mathrm{M}}$ $\approx 179.55 \mathrm{MHz}$. Whereas a purely mechanical response would lead to a symmetric Lorentzian peak, the observed peak is asymmetric, indicating a small contribution from stray electronic transmission in the sample holder (see supplementary material). To extract the mechanical signal strength, we fit these traces with the following function, which takes account of the resonant mechanical response, a non-resonant electrical background, and broadband detection noise (see supplementary material):

$$
P_{\text {out }}(f)=\frac{A^{2}}{Z_{0}}\left|B e^{i \phi_{B}}+\frac{f_{\mathrm{M}}^{2} / Q_{\mathrm{M}}}{f_{\mathrm{M}}^{2}-f^{2}+i \frac{f f_{\mathrm{M}}}{Q_{\mathrm{M}}}}\right|^{2} \delta V_{\mathrm{G}}^{2}+\frac{S_{V V} \Delta f}{Z_{0}},
$$

where $Z_{0}=50 \Omega$ is the line impedance, $Q_{\mathrm{M}}$ is the mechanical quality factor, $S_{V V}$ is the one-sided spectral density of the system voltage noise, $\Delta f$ is the resolution bandwidth, and $A$, $B$, and $\phi_{B}$ are constants. By fitting Fig. 3(c) to Eq. (1), using

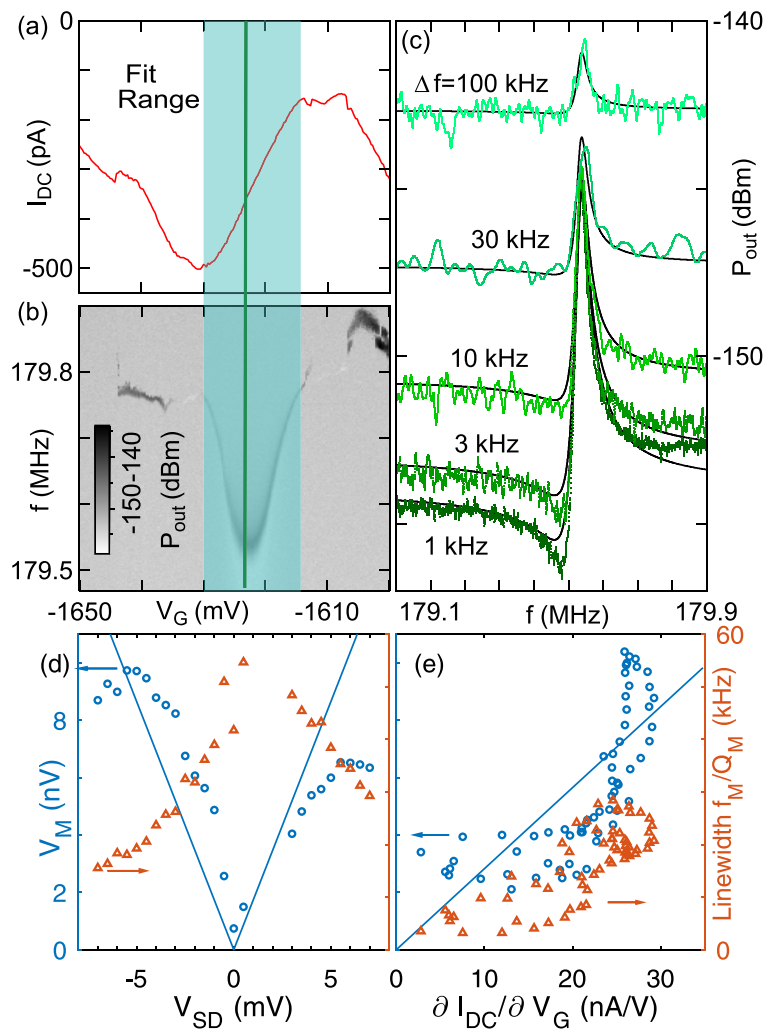

FIG. 3. Measuring the displacement sensitivity (a) DC current as a function of gate voltage in the region of one Coulomb blockade peak, with $V_{\mathrm{SD}}$ $=-5 \mathrm{mV}$. (b) Mechanical resonance measured in RF power simultaneously with (a). (c) Transmission as a function of frequency, measured with gate excitation voltage $\delta V_{\mathrm{G}}=1.2 \mu \mathrm{V}$ and for different settings of the network analyzer's resolution bandwidth $\Delta f$. Points: data. Curves: fits to Eq. (1). Measurements are taken on the flank of the Coulomb blockade peak [vertical line in (a) and (b)]. (d) Mechanical signal (circles, left axis) and linewidth (triangles, right axis) as functions of bias. Line: fit of the form $V_{\mathrm{M}} \propto\left|V_{\mathrm{SD}}\right|$. (e) The same quantities as functions of transconductance. Line: fit of the form $V_{\mathrm{M}} \propto \partial I_{\mathrm{DC}} / \partial V_{\mathrm{G}}$.
$f_{\mathrm{M}}, Q_{\mathrm{M}}, S_{V V}, A, B$, and $\phi_{B}$ as global fit parameters, we find a voltage sensitivity $\sqrt{S_{V V}}=50.3 \pm 0.5 \mathrm{pV} / \sqrt{\mathrm{Hz}}$ and a quality factor $Q_{\mathrm{M}}=6000 \pm 1500$. From Fig. 3(b), it is clear that the linewidth varies strongly with gate voltage across the Coulomb peak, which implies that the quality factor is limited by dissipation due to inelastic electron tunneling. 9,10 The fitting function does not take account of possible mechanical non-linearity, which is justified by the good quality of the fits in Fig. 3(c). We have also confirmed that this drive amplitude is below the observable onset of Duffing distortion of the lineshape and is also below the onset of powerbroadening. ${ }^{34}$

On resonance, the purely electromechanical part of the signal in Fig. 3(c) is

$$
\begin{aligned}
V_{\mathrm{M}} & =A \delta V_{\mathrm{G}}, \\
& =8.79 \pm 0.08 \mathrm{nV} .
\end{aligned}
$$

To quantify the sensitivity, we must relate this output voltage amplitude to the corresponding displacement $u$. The displacement depends on the driving force $\delta F$ via

$$
\begin{aligned}
u & =\left|\chi_{\mathrm{M}}(f)\right| \delta F \\
& =\left|\chi_{\mathrm{M}}(f)\right| V_{\mathrm{G}} \frac{\partial C_{2}}{\partial u} \delta V_{\mathrm{G}},
\end{aligned}
$$

where $C_{2}$ is the capacitance between the gate and the nanotube. Here, $\chi_{\mathrm{M}}(f) \equiv \frac{1}{4 \pi^{2} m}\left[f_{\mathrm{M}}^{2}-f^{2}+i \frac{f f_{\mathrm{M}}}{Q_{\mathrm{M}}}\right]^{-1}$ is the mechanical susceptibility $^{35}$ at driving frequency $f$, and $m$ is the mass. The force is $\delta F=V_{\mathrm{G}} \frac{\partial C_{2}}{\partial u} \delta V_{\mathrm{G}}$. We must therefore estimate the parameters in Eq. (5). This is complicated by the uncertainty in the nanotube's mass and diameter and in the suspended height above the gate, which affects the capacitance derivative. The full estimation procedure is described in the supplementary material. Using the known gate capacitance $C_{2} \approx 3.3 \pm 0.6 \mathrm{aF}$, extracted from a Coulomb blockade measurement similar to Fig. 1(b), we use finite-element electrostatic simulation to deduce that the height is $H=18_{-13.5}^{+92} \mathrm{~nm}$. Combining this value with the nanotube diameter $D=4.5 \pm 1.5 \mathrm{~nm}$, estimated from transmission electron microscopy, leads to a capacitance derivative $\partial C_{2} / \partial u$ $=49.4_{-40.5}^{+271} \mathrm{pFm}^{-1}$. The error range is dominated by the uncertainty in the suspended height and suspension angle $\theta$. The nanotube's mass is $m=21.3_{-15.5}^{+81} \mathrm{ag}$, with the uncertainty arising from the unknown number of walls and from the suspension angle $\theta$.

Substituting these quantities into Eq. (5) allows us to calculate that in Fig. 3(c), the mechanical amplitude is $u=25.2_{-24.3}^{+811} \mathrm{pm}$. The proportionality constant between the signal voltage [Eq. (3)] and the corresponding displacement is therefore known, finally leading to a displacement sensitivity, defined as the square root of the measurement imprecision

$$
\begin{aligned}
\sqrt{S_{u u}} & =\frac{u}{V_{M}} \sqrt{S_{V V}}, \\
& =144_{-139}^{+4700} \mathrm{fm} / \sqrt{\mathrm{Hz}} .
\end{aligned}
$$

The large range of this estimate reflects the combination of uncertainties entering Eq. (5). 
In this model, the electromechanical signal $V_{\mathrm{M}}$ should increase with $\left|V_{\mathrm{SD}}\right|$ because a larger bias leads to a larger mechanically modulated current. This is tested in Fig. 3(d), which plots $V_{\mathrm{M}}$ against $V_{\mathrm{SD}}$. As expected, the signal is approximately proportional to bias. At high bias, the signal falls below the trend because the SET Coulomb peaks become less sharp. The electromechanical signal should also be proportional to the SET transconductance $\partial I_{\mathrm{DC}} / \partial V_{\mathrm{G}}$. This is tested in Fig. 3(e), which plots $V_{\mathrm{M}}$ against transconductance at fixed bias. The data show approximate proportionality, again confirming that the signal arises mainly from conductance through the SET.

We now compare the experimental displacement sensitivity with what would be achieved by a quantum-limited detector. ${ }^{25}$ In a continuous phase-preserving measurement, minimum uncertainty requires the imprecision noise to be equal to the noise generated by backaction. In this ideal case, the displacement sensitivity obeys the standard quantum limit $(\mathrm{SQL})^{36,37}$

$$
\begin{aligned}
\sqrt{S_{\text {ии }}(\mathrm{SQL})} & =\sqrt{\frac{\hbar Q_{\mathrm{M}}}{4 \pi^{2} m f_{\mathrm{M}}^{2}}}, \\
& =4.8_{-2.9}^{+5.5} \mathrm{fm} / \sqrt{\mathrm{Hz}} .
\end{aligned}
$$

Comparing Eq. (9) with Eq. (7) gives the normalised sensitivity

$$
\frac{\sqrt{S_{u u}}}{\sqrt{S_{u u}(\mathrm{SQL})}}=30_{-27}^{+440}
$$

meaning that this measurement is within a factor 470 of the SQL. A larger mass than estimated, for example because of surface contamination, would imply smaller sensitivity relative to the SQL; a geometry in which the nanotube sags close to the gate implies a larger sensitivity but still within the range of Eq. (10). The uncertainty could be reduced by calibrating the displacement using a measurement of Brownian motion. Imprecision below the SQL is possible if measurement backaction excites the resonator out of its ground state. $^{37}$

In fact, some backaction is evident in Fig. 3(b), which shows that the gate voltage range with the strongest signal also leads to a broader mechanical resonance. Figure 3(e) confirms this by plotting the mechanical linewidth against transconductance, showing that higher transconductance, and therefore stronger measurement, correlates with a broader line. This is because the fluctuating occupation of the SET creates a stochastic force which reduces the mechanical quality factor. ${ }^{38,39}$ However, the dependence on source-drain bias is opposite [Fig. 3(d)]. Both behaviors have been explained by considering the damping mechanism. ${ }^{40}$ Electromechanical damping occurs when the changing displacement brings the SET's chemical potential alternately above and below the Fermi level in one of the leads so that electrons preferentially tunnel onto the SET with low energy and off with high energy. This damping is reduced by detuning the quantum dot chemical potential away from one or both Fermi levels. In the situation of this measurement, where the bias is large compared to both the thermal energy and lifetime broadening, this detuning is achieved by separating the two Fermi levels with a sourcedrain bias [Fig. 3(d)] or by tuning the SET chemical potential between the Fermi levels with a gate voltage [Fig. 3(e)]. This behaviour has been previously observed and modelled quantitatively. ${ }^{39}$ At zero bias, occupation fluctuations create mechanical backaction without contributing to the signal. Increasing the bias therefore increases the efficiency of the measurement, allowing the SQL to be approached more closely. This description ignores the effect of transport resonances and energy-dependent tunneling, which lead to more complex backaction even including negative damping. ${ }^{40} \mathrm{~A}$ more complete theory of this RF displacement sensor would need to include these effects to assess the potential for the quantum-limited measurement.

Finally, we consider what limits the achieved displacement imprecision given by Eq. (7). The imprecision is approximately as expected given the noise of the cryogenic amplifier (see supplementary material) and could therefore be improved by using an improved superconducting amplifier. ${ }^{41}$ Alternatively, the conversion of displacement to current could be improved with larger DC gate voltage, while increasing the quality factor of the tank circuit could give larger transimpedance. Ultimately, the sensitivity will be limited by the SET's shot noise. ${ }^{42,43}$

In conclusion, this experiment shows how to monitor a vibrating carbon nanotube with low noise and high speed using an integrated SET transducer. Such a device could monitor weak and transient forces on the nanoscale, for example, in scanning probe microscopy. This resonator also approaches the quantum regime in terms of both the ratio of phonon energy to thermal energy (approximately 1:3) and the measurement sensitivity. This work therefore opens the way to measuring dynamic electron-phonon coupling effects.

See supplementary material for details on fabrication, the derivation of Eq. (1), modelling of the impedance matching circuit, and details of how the uncertainties in Eqs. (7)-(10) are calculated.

We acknowledge DSTL, EPSRC (Nos. EP/J015067/1 and EP/N014995/1), TWCF, FQXI, and RAEng. We thank S. Kafanov and A. Romito for comments and A. W. Robertson for microscopy.

${ }^{1}$ V. Sazonova, Y. Yaish, H. Üstünel, D. Roundy, T. A. Arias, and P. L. McEuen, Nature 431, 284 (2004).

${ }^{2}$ A. K. Hüttel, G. A. Steele, B. Witkamp, M. Poot, L. P. Kouwenhoven, and H. S. J. Van Der Zant, Nano Lett. 9, 2547 (2009).

${ }^{3}$ J. Moser, A. Eichler, J. Güttinger, M. I. Dykman, and A. Bachtold, Nat. Nanotechnol. 9, 1007 (2014).

${ }^{4}$ J. Moser, J. Güttinger, A. Eichler, M. J. Esplandiu, D. E. Liu, M. I. Dykman, and A. Bachtold, Nat. Nanotechnol. 8, 493 (2013).

${ }^{5}$ H. Y. Chiu, P. Hung, H. W. C. Postma, and M. Bockrath, Nano Lett. 8, 4342 (2008).

${ }^{6}$ B. Lassagne, D. Garcia-Sanchez, A. Aguasca, and A. Bachtold, Nano Lett. 8, 3735 (2008).

${ }^{7}$ J. Chaste, A. Eichler, J. Moser, G. Ceballos, R. Rurali, and A. Bachtold, Nat. Nanotechnol. 7, 301 (2012).

${ }^{8}$ R. Leturcq, C. Stampfer, K. Inderbitzin, L. Durrer, C. Hierold, E. Mariani, M. Schultz, F. Oppen, and K. Ensslin, Nat. Phys. 5, 327 (2009). 
${ }^{9}$ G. A. Steele, A. K. Hüttel, B. Witkamp, M. Poot, H. B. Meerwaldt, L. P. Kouwenhoven, and H. S. J. van der Zant, Science 325, 1103 (2009).

${ }^{10}$ B. Lassagne, Y. Tarakanov, J. Kinaret, G. S. Daniel, and A. Bachtold, Science 325, 1107 (2009).

${ }^{11}$ A. Benyamini, A. Hamo, S. V. Kusminskiy, F. von Oppen, and S. Ilani, Nat. Phys. 10, 151 (2014)

${ }^{12}$ A. Eichler, J. Moser, J. Chaste, M. Zdrojek, I. Wilson-Rae, and A. Bachtold, Nat. Nanotechnol. 6, 339 (2011).

${ }^{13}$ A. Castellanos-Gomez, H. B. Meerwaldt, W. J. Venstra, H. S. J. van der Zant, and G. A. Steele, Phys. Rev. B 86, 041402 (2012).

${ }^{14}$ M. Ganzhorn, S. Klyatskaya, M. Ruben, and W. Wernsdorfer, ACS Nano 7, 6225 (2013)

${ }^{15}$ K. Willick, C. Haapamaki, and J. Baugh, J. Appl. Phys 115, 114501 (2014).

${ }^{16}$ B. H. Schneider, V. Singh, W. J. Venstra, H. B. Meerwaldt, and G. A. Steele, Nat. Commun. 5, 5819 (2014).

${ }^{17}$ M. Poggio and B. E. Herzog, "Force-detected nuclear magnetic resonance," in Micro and Nano Scale NMR: Technologies and Systems (Wiley, 2018), p. 381.

${ }^{18}$ H. B. Meerwaldt, S. R. Johnston, H. S. J. van der Zant, and G. A. Steele, Appl. Phys. Lett. 103, 053121 (2013).

${ }^{19}$ K. Willick, X. S. Tang, and J. Baugh, Appl. Phys. Lett. 111, 223108 (2017).

${ }^{20}$ S. L. de Bonis, C. Urgell, W. Yang, C. Samanta, A. Noury, J. VergaraCruz, Q. Dong, Y. Jin, and A. Bachtold, Nano Lett. 18, 5324 (2018).

${ }^{21}$ N. Ares, T. Pei, A. Mavalankar, M. Mergenthaler, J. H. Warner, G. A. D. Briggs, and E. A. Laird, Phys. Rev. Lett. 117, 170801 (2016).

${ }^{22}$ Y. Xu, C. Chen, V. V. Deshpande, F. A. Direnno, A. Gondarenko, D. B. Heinz, S. Liu, P. Kim, and J. Hone, Appl. Phys. Lett. 97, 243111 (2010).

${ }^{23}$ J. P. Mathew, R. Patel, A. Borah, C. B. Maliakkal, T. S. Abhilash, and M. M. Deshmukh, Nano Lett. 15, 7621 (2015).

${ }^{24}$ R. G. Knobel and A. N. Cleland, Nature 424, 291 (2003).

${ }^{25}$ M. D. LaHaye, O. Buu, B. Camarota, and K. C. Schwab, Science 304, 74 (2004).
${ }^{26}$ E. A. Laird, F. Pei, W. Tang, G. A. Steele, and L. P. Kouwenhoven, Nano Lett. 12, 193 (2012).

${ }^{27}$ Y. A. Pashkin, T. F. Li, J. P. Pekola, O. Astafiev, D. A. Knyazev, F. Hoehne, H. Im, Y. Nakamura, and J. S. Tsai, Appl. Phys. Lett. 96, 263513 (2010).

${ }^{28}$ N. Ares, F. J. Schupp, A. Mavalankar, G. Rogers, J. Griffiths, G. A. C. Jones, I. Farrer, D. A. Ritchie, C. G. Smith, A. Cottet, G. A. D. Briggs, and E. A. Laird, Phys. Rev. Appl. 5, 034011 (2016).

${ }^{29}$ C. C. Wu, C. H. Liu, and Z. Zhong, Nano Lett. 10, 1032 (2010).

${ }^{30}$ F. Pei, E. A. Laird, G. A. Steele, and L. P. Kouwenhoven, Nat. Nanotechnol. 7, 630 (2012).

${ }^{31}$ A. Mavalankar, T. Pei, E. M. Gauger, J. H. Warner, G. A. D. Briggs, and E. A. Laird, Phys. Rev. B 93, 235428 (2016).

${ }^{32}$ N. Mason, M. J. Biercuk, and C. M. Marcus, Science 303, 655 (2004).

${ }^{33}$ E. A. Laird, F. Kuemmeth, G. A. Steele, K. Grove-Rasmussen, J. Nygård, K. Flensberg, and L. P. Kouwenhoven, Rev. Mod. Phys. 87, 703 (2015).

${ }^{34}$ H. B. Meerwaldt, G. A. Steele, and H. S. Van Der Zant, "Carbon nanotubes: nonlinear high-Q resonators with strong coupling to single-electron tunneling," in Fluctuating Nonlinear Oscillators: From Nanomechanics to Quantum Superconducting Circuits (Oxford University Press, 2012).

${ }^{35}$ M. Aspelmeyer, T. J. Kippenberg, and F. Marquardt, Rev. Mod. Phys. 86, 1391 (2014)

${ }^{36}$ C. A. Regal, J. D. Teufel, and K. W. Lehnert, Nat. Phys. 4, 555 (2008).

${ }^{37}$ A. A. Clerk, M. H. Devoret, S. M. Girvin, F. Marquardt, and R. J. Schoelkopf, Rev. Mod. Phys. 82, 1155 (2010).

${ }^{38}$ A. Naik, O. Buu, M. D. LaHaye, A. D. Armour, A. A. Clerk, M. P. Blencowe, and K. C. Schwab, Nature 443, 193 (2006).

${ }^{39}$ H. B. Meerwaldt, G. Labadze, B. H. Schneider, A. Taspinar, Y. M. Blanter, H. S. Van Der Zant, and G. A. Steele, Phys. Rev. B 86, 115454 (2012).

${ }^{40}$ A. A. Clerk and S. Bennett, New J. Phys. 7, 238 (2005).

${ }^{41}$ M. Mück and R. McDermott, Supercond. Sci. Technol. 23, 093001 (2010).

${ }^{42}$ S. Kafanov and P. Delsing, Phys. Rev. B 80, 155320 (2009).

${ }^{43}$ Y. Wang and F. Pistolesi, Phys. Rev. B 95, 035410 (2017). 